\title{
Native Cardiac Valve Intervention Type
}

National Cancer Institute

\section{Source}

National Cancer Institute. Native Cardiac Valve Intervention Type. NCI Thesaurus. Code C139050.

A description of what, if any, kind of intervention has been performed on a native cardiac valve. 